\title{
Stratégies frugales pour la fabrication d'instruments scientifiques basés sur la photonique à coût abordable
}

\author{
François PIUZZI \\ Groupe Physique pour le développement, Société Européenne de Physique \\ www.physdev.org piuzzifr@gmail.com
}

L'accès à l'instrumentation scientifique est essentiel pour le développement des pays à faibles ressources. II conditionne les possibilités de mesure de paramètres physiques, chimiques et biologiques, en particulier pour la caractérisation des problèmes sociétaux comme la pollution atmosphérique, la pureté de l'eau, l'analyse de médicaments, la détection de maladies, le réchauffement climatique. II est également important pour la formation universitaire en sciences expérimentales car il est indispensable pour la formation des étudiants, techniciens, expérimentateurs et chercheurs ainsi que pour la réalisation d'expériences scientifiques.

'instrumentation scientifique reste chère et nécessite des conditions d'utilisation qui souvent ne sont pas à la portée des pays à faibles ressources, sans évoquer le problème de l'accessibilité aux pièces détachées, des consommables et de l'impossibilité de mesures de terrain.

Cette situation est en train de changer avec l'émergence de nouvelles méthodes: impression 3D, plateforme électronique open-source Arduino (www.arduino.cc), mini ordinateur à bas coût Raspberry $\mathrm{Pi}$, mesures à distance avec des drones, ainsi que de nouvelles pratiques : science collaborative (crowdsourcing), matériel et logiciels en libre accès (hardware et software open source) et le libre accès à des articles scientifiques (comme PLOS, Public Library Of Science). Cette évolution est surtout due à des scientifiques solidaires, à des amateurs éclairés (geeks) ainsi qu'à l'émergence du DIY en science (do ityourself, voir www.instructables.com) qui a amené une croissance importante de la créativité. Le libre accès permet, comme pour des logiciels, d'améliorer petit à petit par la collaboration et le partage les instruments proposés. C'est également dû à une volonté de réaliser des mesures de manière indépendante dans le cas de problèmes sociétaux (Public Lab). En outre, l'évolution de la technologie permet maintenant

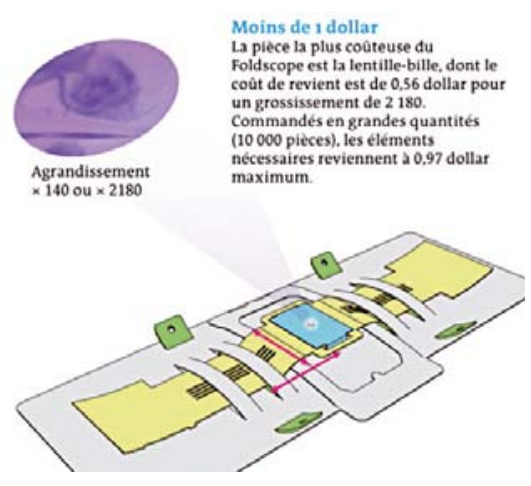

l'accès à des composants de haute technologie (souvent miniaturisés) pour des prix très bas car ils sont utilisés dans des appareils « grand public » notamment en photonique (détecteurs, caméras, LEDs du proche IR à I'UV, capteurs). La conjonction de l'évolution des logiciels, en particulier pour la cartographie, associée à la présence de GPS sur beaucoup d'accessoires permet la réalisation de cartographies à partir de mesures multiples pour caractériser la pollution par exemple (http:// publiclab.org/).

Il est maintenant possible que des scientifiques des pays à faibles ressources, après une formation de quelques mois, puissent concevoir et fabriquer localement des instruments scientifiques qui, s'ils ne sont pas l'équivalent des instruments haut de gamme, permettent néanmoins de faire rentrer ces pays dans le domaine de l'instrumentation scientifique.

La photonique a toujours été partie prenante dans l'instrumentation scientifique car elle permet de maîtriser la lumière, outil de base de la mesure et de la caractérisation. Nous allons présenter des exemples de son utilisation «frugale » dans différents domaines en prenant le cas où l'instrument est conçu totalement de manière frugale, et celui où c'est un accessoire frugal qui est attaché à un équipement à coût plus élevé. Nous parlerons également de l'exemple de l'apport de la « science citoyenne » qui incite des citoyens à faire des mesures pour des scientifiques, ce qui a pour conséquence que le prix des équipements utilisés en grand nombre baisse rapidement.

\section{La microscopie}

Beaucoup de développements d'instruments à coût raisonnable concernent la microscopie car beaucoup d'applications y font appel. Récemment un microscope extrêmement frugal et créatif (coût $<1$ \$) a été développé par Manu Prakask de Stanford [1] : du fait de sa méthode de construction, il a été appelé microscope 

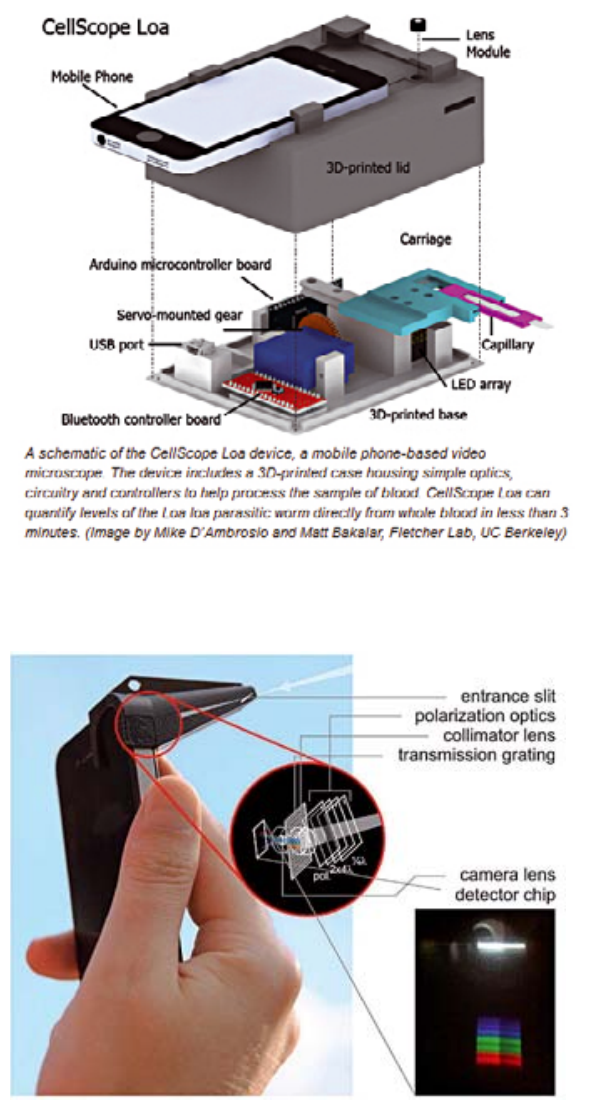

origami ou « Foldscope ». II est constitué de papiers pliés et d'une bille de verre utilisée comme lentille qui permet d'obtenir des grossissements jusqu'à $\times 2180$ [2]

Une autre solution utilisée dans la littérature est l'ajout d'une lentille extérieure à l'appareil photo d'un smartphone. De multiples solutions ont été développées, de la plus simple avec un support de lentille inclus dans une structure imprimée en 3D [3], jusqu'à l'option de lentille inverse [4]. Des applications pour la biologie sont également présentes dans la littérature avec par exemple le suivi en temps réel de la mobilité de cellules [5]. Une initiative de l'université de Berkeley (http://cellscope.berkeley.edu/) appelée Aiding the world with mobile microscopy (aidons le monde par la microscopie mobile), a débouché sur la conception de microscopes liés à des téléphones mobiles pour réaliser des mesures sur le terrain. Les très bonnes performances permettent des applications à la détection de maladies comme la tuberculose et la malaria ainsi que des parasites dans le sang [6], des développements vers la médecine pour les pays à faible ressources comme la détection d'ulcères de la cornée [7]. La structure Cellscope a créé une start up pour les applications en médecine.

\section{La spectrométrie}

De la même manière on trouve beaucoup d'exemples dans la littérature qui utilisent un morceau de DVD comme réseau de diffraction, une webcam comme détecteur et une fibre plastique pour l'entrée, avec des performances tout à fait intéressantes pour des coûts inférieurs à $100 €$ [8] L'impression 3D est utilisée pour réaliser les supports des différents composants ainsi que de l'enveloppe qui intègre les différents éléments constituant le spectromètre (domaine du spectre visible). Cela est très intéressant pour les travaux pratiques à l'université. Il est également possible d'adjoindre une extension (généralement imprimée en 3D) à un smartphone comprenant un réseau de diffraction et permettant d'obtenir directement le spectre sur l'écran. Des projets concernant la spectrométrie Raman sont en cours [9].

Un exemple intéressant est l'initiative « citoyenne » de mesures des aérosols par spectro-polarimétrie réalisées par un réseau de scientifiques citoyens en Hollande en utilisant l'iSPEC (http://ispex. nl/en/), un accessoire que l'on attache à un smartphone. Cela permet en liaison avec des mesures complémentaires de réaliser la cartographie de la concentration d'aérosols dans le pays [10]. La production en masse de l'accessoire permet d'obtenir des coûts très bas.

Des spectromètres frugaux adaptés à la mesure de fluorescence pour la détermination de la nature de l'huile présente dans l'eau de mer ont été développés par la structure Public Lab [11]. La source d'excitation est un pointeur laser UV. Les LED UV sont également des sources de lumière d'excitation de choix pour la fluorescence.

\section{L'astronomie}

L'approche frugale et le financement participatif ont permis à l'association non gouvernementale Astronomers Without Borders (AWB) de concevoir et fabriquer des télescopes performants et à bas coût comme le modèle ONESKY (200 \$) qui a été très bien noté par la revue Sky and Telescopes [12].

\section{L’océanographie}

Le projet européen citoyen Citclops [13] a réalisé des capteurs (KdUINO) pour mesurer le coefficient d'atténuation diffuse Kd relié à la transparence de l'eau des mers. Les capteurs sont accrochés à une bouée et ils transforment l'irradiance au niveau du capteur en impulsions de différentes fréquences. Les informations traitées sont ensuite transférées au bateau le plus proche.

Les stratégies frugales dont nous vous avons décrit ici quelques exemples permettent la conception et la réalisation $d$ 'instruments scientifiques performants et à un coût abordable en particulier pour les pays à faible ressources. Les méthodes mises en œuvre à l'origine dans ce but sont maintenant également utilisées dans les
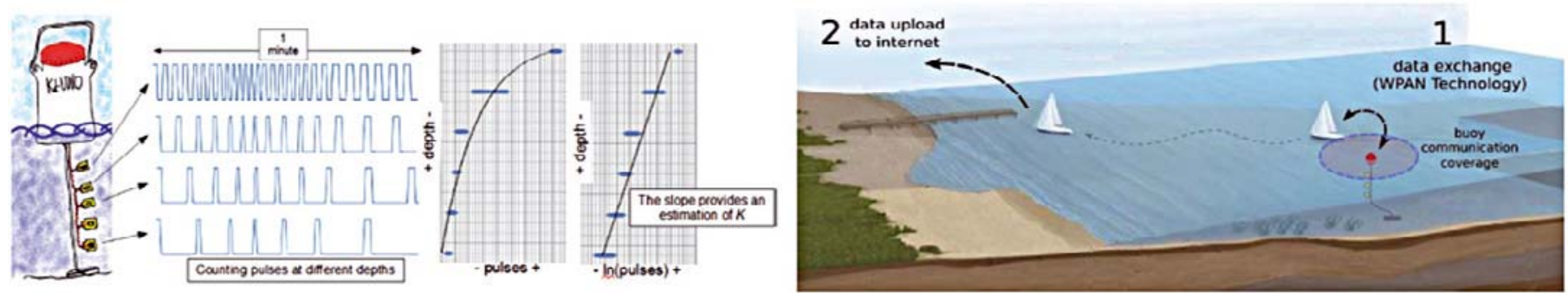
milieux industriels. La principale qualité de ces méthodes est qu'elles permettent à la créativité des scientifiques (et citoyens avertis) de s'exprimer facilement.

Nous voudrions souligner l'apport important de l'impression 3D pour tous ces développements. Cette technique permet de réaliser des éléments importants pour l'utilisation de l'optique comme les supports de lentilles, de miroirs, des rails, supports de pointeurs laser, etc. Elle est d'autant plus importante pour les universités des pays à faibles ressources qui ne disposent pas d'ateliers de mécanique. Les fichiers 3D pour la plupart de ces éléments se trouvent en libre accès par exemple sur http://www.thingiverse.com/.

Pour une introduction aux stratégies frugales, on peut aussi consulter le livre de Josuah Pearce Open-Source Lab: How to Build Your Own Hardware and Reduce Research Costs (Elsevier, 2014). Les Fab Labs, dont le développement en France est récent, constituent une bonne opportunité pour se former à l'impression $3 \mathrm{D}$ et au prototypage rapide.

II faut rappeler que la plupart des développements que nous avons cités ici ont été réalisés sous la licence CERN Open Hardware License 1.1 et les documents sous Creative Commons AttributionShareAlike 3.0 Unported License.

\section{Pour en savoir plus}

[1] http://journals.plos.org/plosone/article?id=10.1371//journal.pone.0098781

[2] http://abonnes.lemonde.fr/sciences/infographie/2014/04/01/le-foldscope-unmicroscope-origami-a-moins-de-1-dollar_4393821_1650684.htmlextmc=microscope_origami\&xtcr=1

[3] http://www.thingiverse.com/thing:280004

[4] http://www.ncbi.nlm.nih.gov/pmc/articles/PMC4031072/pdf/pone.0095330.pdf

[5] http://journals.plos.org/plosone/article?id=10.1371//journal.pone.0103547

[6] http://newscenter.berkeley.edu/2015/05/06/video-cellscope-automates-detection-of-parasites/

[7] R.N. Maamari, S. Ausayakhun, T.P. Margolis, D.A. Fletcher, J.D. Keenan, Novel telemedicine device for diagnosis of corneal abrasions and ulcers in resource-poor settings, JAMA Ophthalmol 132, 894-895 (2014)

[8] http://store.publiclab.org/products/desktop-spectrometry-kit-3-0

[9] http://hackaday.io/project/1279-ramanpi-raman-spectrometer

[10] http://onlinelibrary.wiley.com/doi/10.1002/2014GL061462/pdf

[1 1 1] http://publiclab.org/wiki/oil-testing-kit

[12] http://www.skyandtelescope.com/wp-content/uploads/OneSkyReflector.pdf

[13] http://www.citclops.eu/media/citclops/org/documents/publications/rbardaji_smallheader.pdf

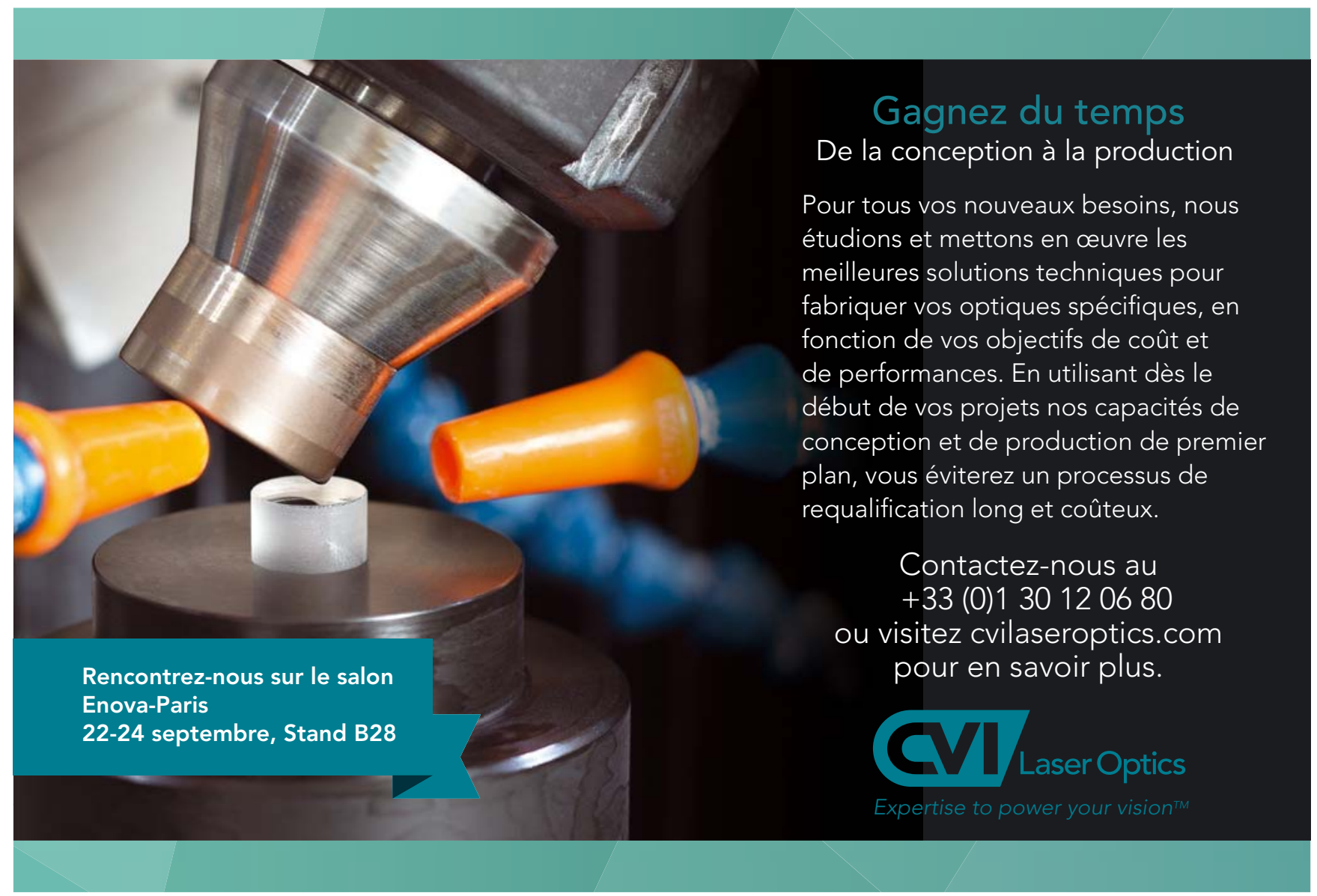

